\title{
Microarray-based analysis of stress-regulated microRNAs in Arabidopsis thaliana
}

\author{
HAN-HUA LIU, XIN TIAN, YAN-JIE LI, CHANG-AI WU, and CHENG-CHAO ZHENG \\ State Key Laboratory of Crop Biology, College of Life Sciences, Shandong Agricultural University, Tai'an, \\ Shandong 271018, People's Republic of China
}

\begin{abstract}
High-salinity, drought, and low temperature are three common environmental stress factors that seriously influence plant growth and development worldwide. Recently, microRNAs (miRNAs) have emerged as a class of gene expression regulators that have also been linked to stress responses. However, the relationship between miRNA expression and stress responses is just beginning to be explored. Here, we identified 14 stress-inducible miRNAs using microarray data in which the effects of three abiotic stresses were surveyed in Arabidopsis thaliana. Among them, 10 high-salinity-, four drought-, and 10 cold-regulated miRNAs were detected, respectively. miR168, miR171, and miR396 responded to all of the stresses. Expression profiling by RT-PCR analysis showed great cross-talk among the high-salinity, drought, and cold stress signaling pathways. The existence of stress-related elements in miRNA promoter regions provided further evidence supporting our results. These findings extend the current view about miRNA as ubiquitous regulators under stress conditions.
\end{abstract}

Keywords: microRNA; microarray; stress; gene expression regulators; Arabidopsis thaliana

\section{INTRODUCTION}

MicroRNAs (miRNAs) are a highly conserved class of small noncoding RNAs that regulate gene expression by posttranscriptional degradation or translational repression (Carrington and Ambros 2003; Bartel 2004). With the discovery of large numbers of miRNAs in both plants and animals, the important roles of these special small RNAs have been widely recognized (Llave et al. 2002; Park et al. 2002; Ambros 2004). Many processes, such as leaf development, auxin signaling, phase transition, flowering, and genome maintenance, are regulated in similar ways by different miRNAs (Aukerman and Sakai 2003; Palatnik et al. 2003; Vaucheret et al. 2004; Guo et al. 2005; Mallory et al. 2005).

Recently, there has been strong evidence leading to the proposal that miRNAs are hypersensitive to abiotic or biotic stress as well as to diverse physiological processes

Reprint requests to: Cheng-Chao Zheng, State Key Laboratory of Crop Biology, College of Life Sciences, Shandong Agricultural University, Tai'an, Shandong 271018, People's Republic of China; e-mail: cczheng@ sdau.edu.cn; fax: +86 538-822 6399; or Chang-Ai Wu, State Key Laboratory of Crop Biology, College of Life Sciences, Shandong Agricultural University, Tai'an, Shandong 271018, People's Republic of China; e-mail: cawu@sdau.edu.cn; fax: +86 538-822 6399.

Article published online ahead of print. Article and publication date are at http://www.rnajournal.org/cgi/doi/10.1261/rna.895308.
(Sunkar and Zhu 2004; Lu et al. 2005). The first report linking miRNA and stress tolerance was miR398, expression of which is transcriptionally down-regulated by oxidative stresses. In Arabidopsis, miR398 was found to target two closely related $\mathrm{Cu} / \mathrm{Zn}$ superoxide dismutase coding genes, cytosolic CSD1 and chloroplastic CSD2, and a reduced level of miR398 led to improved tolerance of transgenic lines compared with the wild-type plants under oxidative stress conditions (Sunkar et al. 2006). Additionally, miR395 and miR399 were identified to be involved in sulfate and inorganic phosphate starvation responses, respectively (Jones-Rhoades and Bartel 2004; Fujii et al. 2005). In rice, miR169g was confirmed as the only member induced by drought among the miR169 family (Zhao et al. 2007). Furthermore, in line with the hypothesis of miRNA's response to environmental stimuli, 21 miRNAs belonging to 11 miRNA families in Arabidopsis were predicted to be upregulated under UV-B stress condition (Zhou et al. 2007). However, systematic expression analysis for miRNAs under abiotic stress conditions in Arabidopsis has not been reported. Hence, efforts to identify novel stress-regulated miRNAs and determine their expression patterns could improve our understanding of their functions in stress adaptation.

Currently, a variety of biochemical, molecular, and bioinformatic approaches and technologies have been developed for miRNA analysis and detection (Schena et al. 1995; Eisen 
and Brown 1999). Using tiling path microarray analysis as a tool, it is now possible to perform high-throughput profiling of the expression of all the known miRNAs to examine their expression profiles under different environmental stresses (Garzon et al. 2006; Zhao et al. 2007). In this work, we analyzed the effects of 117 miRNAs under highsalinity, drought, and low-temperature stress conditions with miRNA chips representing nearly all known miRNAs cloned or identified in Arabidopsis. Fourteen stress-inducible miRNAs were detected, and our results were further confirmed by detecting their expression patterns and analyzing the cis-elements in their promoter sequences.

\section{RESULTS AND DISCUSSION}

\section{Arabidopsis miRNA microarray experiments}

In order to test whether the expression of any of the currently known miRNAs was regulated by abiotic stresses, we prepared a miRNA microarray containing 117 probes that were complementary to known Arabidopsis miRNAs. In addition, eight short oligos possessing no homology with any existing miRNA sequences were designed as negative controls, and a transcriptional repressor Hex as positive control was also included. Total RNA was extracted from the whole plants, and low-molecular-weight miRNAs, which were used for preparation of labeled probes, were obtained. Microarrays were hybridized with Cy3 probe pairs of high-salinity-, drought-, and cold-treated plants and unstressed plants as described in Materials and Methods. The hybridized microarray was scanned by a separate laser channel for $\mathrm{Cy} 3$ emissions. The ratio of the treated and untreated plants' fluorescent signal intensities was then measured as a relative measure to determine changes in the differential expression of the oligomer sequence represented by miRNA spots on the microarrays. The quality of the microarray data was assessed, and standard quality-control measures indicated that the microarray used in this analysis showed a similar distribution of intensities. None of the negative control probes gave a detectable signal in any of the samples. The $q$ value calculated from different samples was used as a measure of biological reproducibility, and for each gene, it was the lowest false discovery rate at which that gene was called significant.

\section{Identification of stress-response miRNAs}

The miRNA populations from different stress treatment samples were compared to a mock sample. Significance analysis of microarrays (SAM) and a criterion of fold change $>1.5$ and $q$ value $<0.001$ were used to examine the effects of various stress treatments. We found that 14 miRNAs (miR156, miR159, miR165, miR167, miR168, miR169, miR171, miR172, miR319, miR393, miR394, miR396, miR397, and miR408) on the microarray showed differential expression profiles in response to abiotic stresses (Table 1). No statistically significantly down-regulated miRNAs were found during this process. Among them, 10 high-salinity-, four drought-, and 10 cold-regulated miRNAs were detected (Fig. 1). miR165, miR319, and miR393 were up-regulated by both high salinity and cold, miR167 was induced by both high salinity and drought, and miR168, miR171, and miR396 were observed to respond remarkably to all three stresses.

Based on the characteristic of their targets, the identified miRNAs could be classified into three major categories (Table 2). The first category includes eight miRNAsmiR156, miR159, miR165, miR169, miR171, miR172, miR319, and miR396- which target transcription factors involved in further regulation of gene expression and signal transduction that probably function in stress responses (Jones-Rhoades and Bartel 2004; Sunkar and Zhu 2004). Thus, it appears that the induction of these miRNAs would lead to the repression of some corresponding transcription factor genes which, in turn, target a set of specific protein coding genes and play defensive roles against stresses. The second category includes miR167, miR168, miR393, and miR394, which are related to direct response to stresses or

TABLE 1. Stress-response miRNAs identified by microarray analysis

\begin{tabular}{|c|c|c|}
\hline Treatment & miRNAs & Fold change \\
\hline \multirow[t]{12}{*}{$300 \mathrm{mM} \mathrm{NaCl}$} & miR396 & 2.99 \\
\hline & miR168 & 1.91 \\
\hline & miR167 & 1.87 \\
\hline & miR165 & 1.82 \\
\hline & miR319 & 1.81 \\
\hline & miR159 & 1.63 \\
\hline & miR394 & 1.58 \\
\hline & miR156 & 1.56 \\
\hline & miR393 & 1.55 \\
\hline & miR171 & 1.50 \\
\hline & miR158 & 1.46 \\
\hline & miR169 & 1.30 \\
\hline \multirow[t]{7}{*}{200 mM mannitol } & miR396 & 2.59 \\
\hline & miR168 & 1.91 \\
\hline & miR167 & 1.84 \\
\hline & $\operatorname{miR} 408$ & 1.73 \\
\hline & miR171 & 1.55 \\
\hline & miR157 & 1.42 \\
\hline & miR393 & 1.36 \\
\hline \multirow[t]{11}{*}{$4^{\circ} \mathrm{C}$} & miR396 & 3.95 \\
\hline & miR397 & 2.08 \\
\hline & $\operatorname{miR} 172$ & 1.88 \\
\hline & miR169 & 1.79 \\
\hline & miR408 & 1.73 \\
\hline & miR168 & 1.71 \\
\hline & miR171 & 1.60 \\
\hline & miR393 & 1.56 \\
\hline & miR319 & 1.54 \\
\hline & mIR165 & 1.50 \\
\hline & miR400 & 1.36 \\
\hline
\end{tabular}




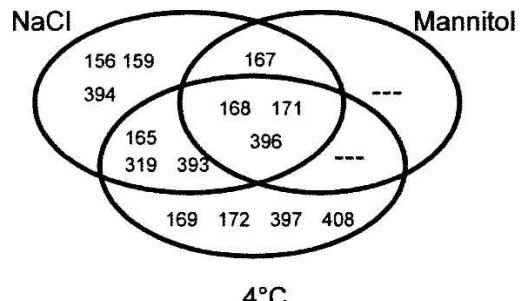

FIGURE 1. Venn diagram illustrates common and unique differential miRNAs expression under three treatment conditions.

external stimuli. miR393 and miR394 target the messages of F-box proteins, which were recently reported to be differentially regulated by stress conditions and play significant roles in the abiotic stress-response pathway (Jones-Rhoades and Bartel 2004; Navarro et al. 2006; Jain et al. 2007). miR168 has been proven to target mRNA of ARGONAUTE 1 (AGO1), which might be the only member of the AGO family that is regulated by a miRNA (Vazquez et al. 2004). The direct role of AGO in plant abiotic stresses has not yet been demonstrated. However, one of its associated partners, Hsp90, is stress-sensitive (Tahbaz et al. 2001; Liu et al. 2005; Leung and Sharp 2007). miR167 targets two auxin response factors, ARF6 and ARF8 (Wu et al. 2006). Plant auxin regulates many agronomically important aspects of plant growth and development as well as responses to environmental stress conditions (Fedoroff 2002; Himmelbach et al. 2003; Achard et al. 2006). Hence, miR167 might play a potential role in stress-resistance progress by affecting auxin signaling pathways. The last category includes miR397 and miR408, whose target genes are hydrolase and oxidoreductase coding genes, which respond to many stresses (Kimura et al. 2003; Apel and Hirt 2004). However, a few of the miRNAs reported to be regulated by high salinity, drought, or cold stress were missed in our results, such as miR389 and miR400 (Sunkar and Zhu 2004). We speculate that this might have been caused by the differences between our treatment methods and theirs or by the technical differences between RNA gel blot and microarray analysis. Some other miRNAs failed to be detected, perhaps because they are required at low levels at this stage or their expression is limited to specific cell types or particular growth conditions, which make it difficult to identify the changes under stress conditions.

In addition, most miRNAs were proven to be involved in the regulation of important developmental processes as shown in Table 2. Stressed plants often resemble abnormal developmental phenotypes, which can be viewed as entering a particular developmental phase (Cooper et al. 2003; Sunkar et al. 2007). Furthermore, there is evidence that many of the miRNA target genes are involved in both development and stress regulation, so we speculate that these miRNAs might be co-regulated by both environmental factors and developmental cues.

\section{Expression profiles of stress-inducible miRNAs during stress treatments}

In microarray data, we found that miRNAs belonging to the same family have similar expression patterns, suggesting that they could not be differentiated in microarray analysis because of a potential cross-hybridization problem. To determine which locus is responsive to stress conditions, we performed semiquantitative RT-PCR analysis using specific primers designed to amplify fold-back precursor transcripts in Arabidopsis. Two-week-old wild-type Arabidopsis seedlings were subjected to stress treatments of

TABLE 2. Putative target genes of stress-inducible miRNAs and their function annotations

\begin{tabular}{lll}
\hline miRNAs & \multicolumn{1}{c}{ Targets } & \multicolumn{1}{c}{ Function description } \\
\hline miR156 & SBP family of transcription factors & Vegetative phase change; root development \\
miR159 & MYB and TCP transcription factors & SD flowering time; anther development leaf \\
miR165 & Class III HD-ZIP transcription factors & Development \\
miR167 & ARF6 and ARF8 & Gynoecium and stamen development \\
miR168 & ARGONAUTE1 & Plant development \\
miR169 & CBF & Floral development \\
miR171 & SCL transcription factors & Flowering time; floral organ identity \\
miR172 & AP2 transcription factors & Morphogenesis of shoot lateral organs \\
miR319 & TCP transcription factors & Bacterial disease resistance \\
miR393 & F-box protein; bHLH transcription factors & - \\
miR394 & F-box protein & - \\
miR396 & GRL transcription factors; Rhodenase-like protein; & - \\
miR397 & Kinesin-like protein B & - \\
miR408 & Laccases; $\beta-6$ tubulin &
\end{tabular}

SBP, squamosa promoter binding protein; SD, short day; TCP, TEOSINTE BRANCHED1, CYCLOIDEA, and PCF; HD-ZIP, class III homeodomain-leucine zipper; ARF, auxin response factors; CBF, CAAT binding factor; SCL, scarecrow-like; AP2, APETALA2; bHLH, basic helix-loop-helix; GRL, growth regulating factor; 
$300 \mathrm{mM} \mathrm{NaCl}, 200 \mathrm{mM}$ mannitol, or $4^{\circ} \mathrm{C}$, respectively. To ensure the RT-PCR was accurate, elongation factor $1-\alpha$ (ef1- $\alpha)$ gene was used as internal control, and varying PCR cycle numbers were determined to quantitatively amplify each transcript (Fig. 2). The RT-PCR results were ultimately consistent with the microarray data for all miRNAs tested except miR156h and miR167d. The induction of miR393 reported here was in agreement with previous studies using Northern blotting (Sunkar and Zhu 2004).

To further analyze the temporal expression patterns of these miRNAs, time course analysis by RT-PCR was performed to detect whether they can be regulated by stress after an extended treatment time (Fig. 3). Our data showed that the levels of miR156h, miR167a, miR167c, miR167d, miR168, and miR171b were gradually increased from 2 to $24 \mathrm{~h}$ after exposure to high-salinity treatment, while expression of miR396a peaked at $24 \mathrm{~h}$. miR167a levels accumulated after $2 \mathrm{~h}$ of drought stress and were greatly increased after $24 \mathrm{~h}$ of treatment, while miR168 first increased then returned to a normal level after $6 \mathrm{~h}$. During cold-stress treatment, most miRNA levels were higher after $6 \mathrm{~h}$ and declined with longer stress; the exception was miR156a, which had maximal expression at $24 \mathrm{~h}$. The expression of additional members of miRNA families was also analyzed, but these transcripts were undetectable or did not change (data not shown). Members of the same miRNA family might have different functional roles in spite of their high similarity in the sequence. Taken together, the newly identified stress-response miRNAs were prone to being induced by all treatments, indicating the existence of great cross talk between high-salinity, drought, and cold stress signaling processes. Our results revealed that a single

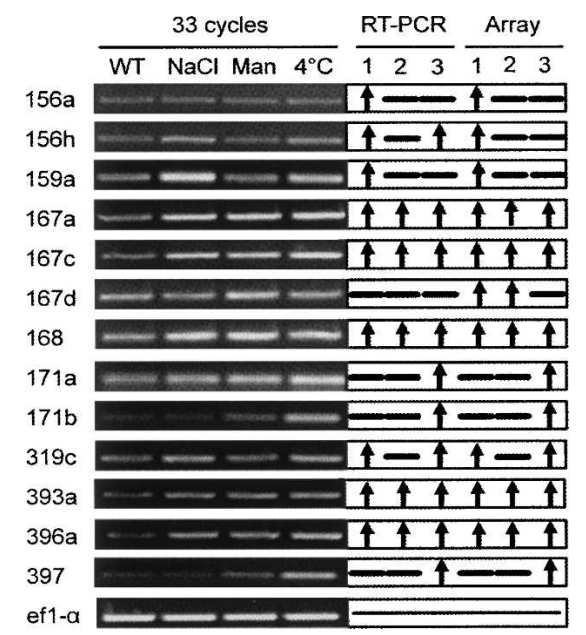

FIGURE 2. RT-PCR validation of the microarray. (RT-PCR) results from RT-PCR analysis; (Array) results from microarray; (Man) mannitol treatment; (lane 1) $300 \mathrm{mM} \mathrm{NaCl}$ treatment; (lane 2) 200 $\mathrm{mM}$ mannitol treatment; (lane 3) $4^{\circ} \mathrm{C}$ treatment; $(\uparrow$ ) up-regulated compared to WT; and (-) no significant difference.
miRNA has the potential to regulate multiple functionally related mRNAs in response to stress.

\section{Stress-relevant cis-elements exist in the promoters of miRNA genes}

Cis- and trans-acting elements involved in stress-induced gene expression have been analyzed extensively (JagloOttosen et al. 1998; Kasuga et al. 1999; Zhang et al. 2005). To further elucidate the inducibility of these products, we analyzed the 1000-base-pair (bp) upstream promoter sequence of 20 miRNAs by using the PlantCARE database (http://intra.psb.ugent.be:8080/PlantCARE) (Lescot et al. 2002). Among the elements listed in Table 3, we identified several known stress-responsive elements, such as the ABA-response elements (ABREs), anaerobic induction elements (AREs), MYB binding site involved in droughtinducibility (MBS), heat-stress-responsive elements (HSEs), low-temperature-responsive elements (LTRs), and defenseand stress-responsive elements (TC-rich repeats). Some other regulatory elements were also identified, such as those possibly involved in regulation in response to gibberellic acid (GA), ethylene, salicylic acid (SA), or methyl jasmonate (MeJA). In plants, most ABA-responsive genes have the conserved ABREs in their promoters, which are significant cis-elements for genes responsive to abiotic stress in Arabidopsis (Mundy et al. 1990; Xu et al. 1996). Of the 20 miRNAs analyzed, six had ABREs, suggesting these miRNAs might be involved in ABA-mediated stress-response processes. Sixteen miRNAs had AREs, which respond to hypoxic, low-temperature, and dehydration stress (Dolferus et al. 1994). The presence of ABREs and AREs suggests that these miRNA might be regulated by stress conditions as protein coding genes. For example, miR393 was reported to be strongly up-regulated by cold, dehydration, and $\mathrm{NaCl}$ treatments. We found seven AREs and two ABREs in the promoters of three miR393 family members (miR393a, -b, and $-\mathrm{c}$ ), which might provide the evidence of the induction of this miRNA. Likewise, the responses of miR167, miR168, and miR396 to high-salinity, drought, and cold treatments are correlated with the enrichment of AREs and ABREs in their promoters. Stress-related elements also existed in miR408 promoters and the role of this miRNA needs to be determined experimentally. Taken together, analysis of stress-response enrichment cis-elements provides additional evidence that these 14 miRNA genes are very likely to be involved in the responses to abiotic stresses.

In conclusion, we analyzed 117 miRNAs expression profiles under three abiotic stress conditions in Arabidopsis and found that 14 of them were differentially regulated by one or more stress conditions. Information about miRNA expression patterns and the biochemical function of their targets is essential and provides a valuable contribution toward our understanding of the function of these tiny noncoding genes and their cooperation in complex biological 

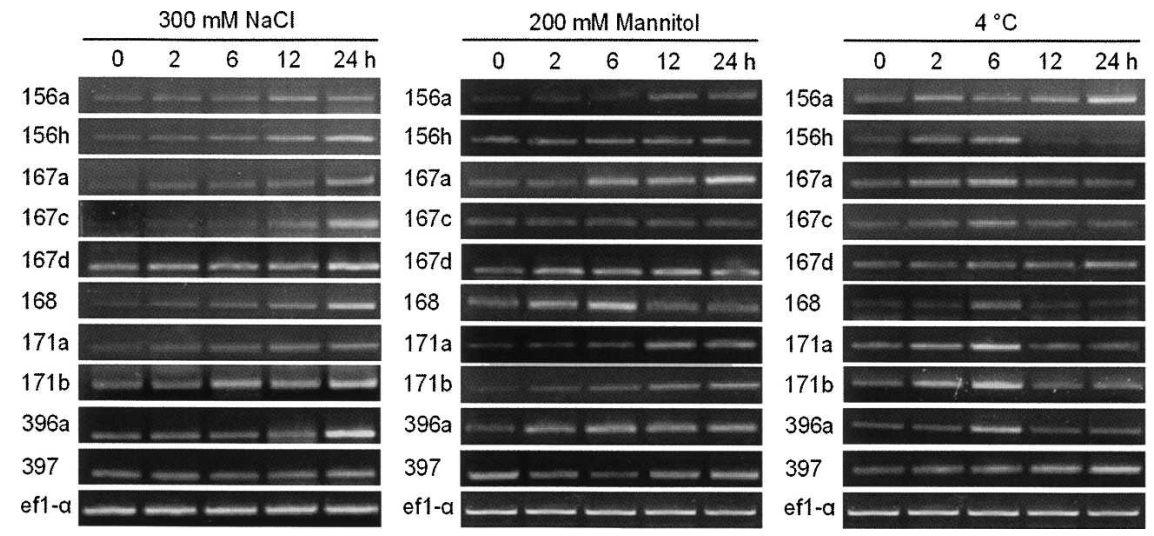

\section{RNA extraction and miRNA microarray experiments}

Total RNA was isolated from different Arabidopsis seedlings with TRIZOL reagent (Invitrogen) and the low-molecular-weight RNA was isolated using the miRNA Isolation Kit (Ambion). Target RNA labeling was performed as described (Thomson et al. 2004). In brief, $4 \mu \mathrm{g}$ of low-molecularweight RNA was labeled with $500 \mathrm{ng}$ of 5' -phosphate-cytidyl-uridyl-cy3-3' (Dharmacon) with 2 units of T4 RNA ligase (NEB). The labeling reaction was performed at $4^{\circ} \mathrm{C}$ for $2 \mathrm{~h}$. Labeled RNA was precipitated with $0.3 \mathrm{M}$ sodium acetate and 2.5 volumes of ethanol and was then resuspended in $15 \mu \mathrm{L}$ of hybridization buffer containing $3 \times$ SSC, $0.2 \%$ SDS, and $15 \%$ formamide. Hybridiza-

networks. Based on the RT-PCR and promoter analysis, we speculate that miR167, miR168, and miR396 might play important roles in plant abiotic stress responses. However, due to the potential uncertainty in this experiment, it is not feasible to just estimate the exact effects of these miRNAs in plants. These data provide a starting point for future studies, and continued efforts are needed to confirm the function of miRNAs in stress response and stress adaptation.

\section{MATERIALS AND METHODS}

\section{Plant materials}

A. thaliana ecotype Columbia seeds were surface-sterilized and sown on MS-agar plates. Seeds were stratified at $4{ }^{\circ} \mathrm{C}$ for $2 \mathrm{~d}$ and then transferred to $22^{\circ} \mathrm{C}$ for $2 \mathrm{wk}$. For different stresses, seedlings were transferred to blotting paper without stress treatment or saturated with $300 \mathrm{mM} \mathrm{NaCl}$, or $200 \mathrm{mM}$ mannitol, or treated with cold $\left(4^{\circ} \mathrm{C}\right)$. For RNA extraction and microarray experiment, the whole plants were frozen and stored in liquid nitrogen immediately after harvest.

\section{MicroRNA microarray construction}

Mature miRNA sequences were downloaded in the miRBase (http://microrna.sanger.ac.uk/, as of June 2005). There were 117 miRNAs from Arabidopsis after redundant sequences were discarded. In addition, eight short oligos were designed to possess no homology with any existing RNA sequence, and their corresponding synthetic miRNAs were produced by in vitro transcription using a miRNA Probe Construction Kit (Ambion). Various amounts of synthetic miRNAs were spiked into the RNA samples. All miRNA probe sequences were designed to be complementary to the full-length mature miRNA. To facilitate probe immobilization on aldehyde-modified glass slides, the probe sequences were concatenated up to $40 \mathrm{nt}$ and modified with $5^{\prime}$-aminomodifier C6. Oligonucleotide probes were synthesized at MWG Biotech and dissolved in EasyArray spotting solution at $40 \mu \mathrm{M}$ concentration. Each probe was printed in triplicate using a SmartArray microarrayer (CapitalBio Corporation). tion was performed using LifterSlip (Erie), which allowed for even dispersal of hybridization solutions between the microarray and coverslip. The hybridization chamber was laid on a three-phase tiling agitator (BioMixer II) to prompt the microfluidic circulation under the coverslip. Hybridization was performed in a water bath at $42^{\circ} \mathrm{C}$ overnight. After the array had been hybridized at $42^{\circ} \mathrm{C}$ overnight, it was washed with two consecutive washing solutions $\left(0.2 \%\right.$ SDS, $2 \times$ SSC at $42^{\circ} \mathrm{C}$ for $5 \mathrm{~min}$, and $0.2 \%$ SSC for $5 \mathrm{~min}$ at room temperature).

\section{Image acquisition and data processing}

Clustering arrays were scanned with a confocal LuxScan scanner. The scanning setting was adjusted to obtain a visualized equal intensity of U6 spots across arrays. Data was extracted from the TIFF images using LuxScan 3.0 software (CapitalBio). Lowintensity spots were removed for which fewer than $30 \%$ of the signal pixels exceeded the median background plus two times its standard deviation. Then normalization was performed based on the mean array intensity for inter-array comparison. For each sample, consideration of two hybridizations was carried out and each miRNA probe had three replicate spots on a microarray. The mean intensity value of each probe was used for cluster analysis. The raw data were Log 2 transformed and median centered by arrays and genes using the Adjust Data function of CLUSTER 3.0 software and then further analyzed with hierarchical clustering with average linkage (Eisen et al. 1998). Original microarray data are deposited at the Gene Expression Omnibus. To determine the significant differentially expressed miRNAs, Significance Analysis of Microarrays (SAM, version 2.1) was performed using two-class unpaired comparison in the SAM procedure (Tusher et al. 2001).

\section{RT-PCR analysis}

Total RNA isolated with RNase Plant Mini Kit (Qiagen) was processed. Reverse transcription reactions were performed in $20 \mu \mathrm{L}$ using $5 \mu \mathrm{g}$ of RNA by M-MLV (NEB). RT-PCR conditions for elongation factor $1-\alpha$ gene ef1- $\alpha$ amplification were as follows: $94^{\circ} \mathrm{C}$ for $5 \mathrm{~min}, 94^{\circ} \mathrm{C}$ for $30 \mathrm{sec}, 55^{\circ} \mathrm{C}$ for $30 \mathrm{sec}, 72^{\circ} \mathrm{C}$ for $1 \mathrm{~min}$, and $72^{\circ} \mathrm{C}$ for $5 \mathrm{~min}, 25$ cycles. Primers for efl- $\alpha$ were as follows: forward, 5' -GTATGGTTGTTACCTTTGCTCCCACAG and reverse, 


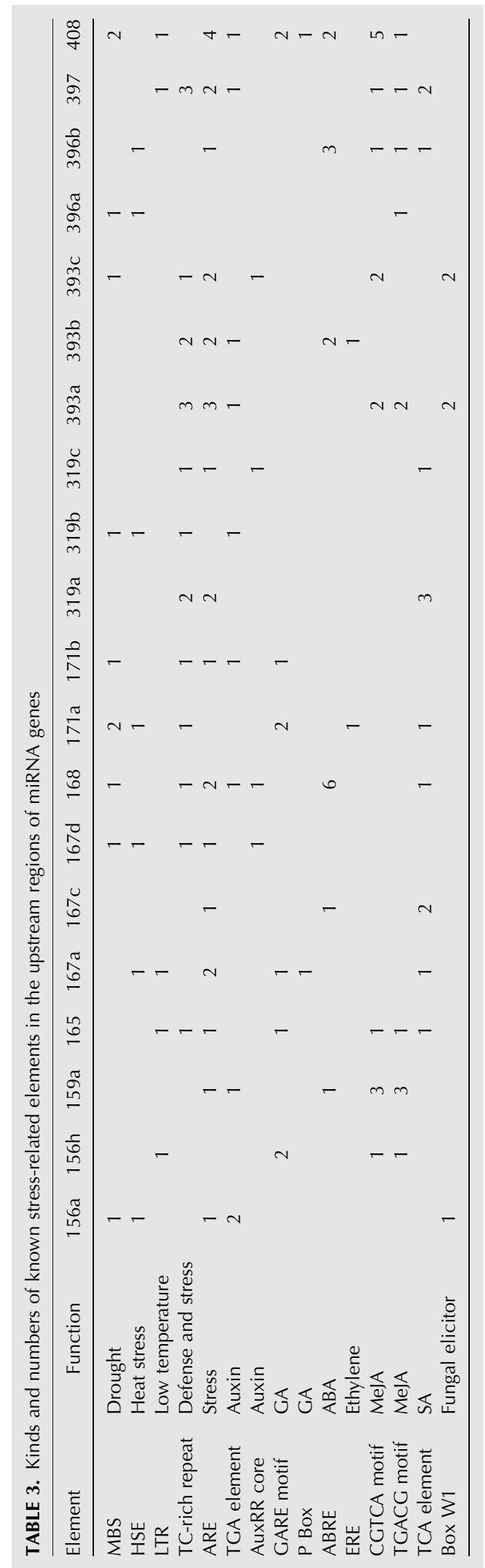


3'-CATCATTTGGCACCCTTCTTCACTGC (500 bp). For miRNA precursor amplification, essentially the same conditions were used except the number of PCR cycles was increased to 33. The primer pairs used for RT-PCR and predicted amplicon sizes were as follows:

156a, 5' -AGAATTTGGTATGCAGAGACAGAT; 3' -CGGTTTCTG GACTAATTGGAA (298 bp);

156h, 5'-TGACACGATCACACAACATGG; 3'-CCACCGTCACT GCTTACTTA (209 bp);

159a, 5' -CACGCTAAACATTGCTTCGGAAT; 3'-ATCCCATAAG CCCTAATCCTTGT (290 bp);

159c, 5'-ACCAAGTTTTGAAGAACAGAGACT; 3'-CATAGAGA GTGCGCGGTGTT (169 bp);

167a， 5'-GTGTAGTCAACTGTGTGCGTT; 3'-GCACAACTTGT TGCTCAGGT (234 bp);

167c, 5' -TTCATGCTACAATCATTAGCAGGT; 3'-AGTCGTCTT CATGTCTGTATGT (205 bp);

167d, 5'-GAGTTGTGGCCATTAAGAGCT; 3'-CTTCTTGTTAAT GTTTGCTCTCTCCT (216 bp);

168, 5'-TGATAGTAGAGTCTCACCATCG; 3'-GAAGGAGAAGC GTAGAAATCTTC (205 bp);

171a, 5' -TCCAAAATAGAGACGAGAGAGT; 3'-CTCCTCCTCAC ACTTCACAT (215 bp);

171b, 5'-CGAGTGCCTGTAGAGTAAAAAC; 3'-TTCTGGAGCT AAGTGGAGATT (278 bp);

319c, 5'-AAACACTCGTGGTAGAGAAACGAT; 3'-AGAGGTTG AAAATGCAAATCCAGT (238 bp);

393, 5'-CAAAGAGATAGCATGATCCAA; 3'-AAGAGGAACACG ATCCATTGAC (214 bp);

396a, 5' -AGGGTTTCGTCTGCTCTACAT; 3'-TCTGATTATGGA ATCAATCACGCT (242 bp);

397, 5' -CCCCTGGGTTTGAATGAACAT; 3'-AGAACTCTCAAG GTCTTTTAAGTG (180 bp).

\section{ACKNOWLEDGMENTS}

We thank Dr. Jianqing Zhao (CapitalBio, Beijing, China) for his technical help. This work was supported by the National Basic Research Program (Grant 2006CB1001006), Program for Changjiang Scholars and Innovative Research Team in University (Grant IRT0635), and the National Natural Science Foundation (Grants 30570144 and 30500042) in China.

Received October 29, 2007; accepted January 24, 2008.

\section{REFERENCES}

Achard, P., Cheng, H., De Grauwe, L., Decat, J., Schoutteten, H., Moritz, T., Van Der Straeten, D., Peng, J., and Harberd, N.P. 2006. Integration of plant responses to environmentally activated phytohormonal signals. Science 311: 91-94.

Ambros, V. 2004. The functions of animal microRNAs. Nature 431: $350-355$.

Apel, K. and Hirt, H. 2004. Reactive oxygen species: Metabolism, oxidative stress, and signal transduction. Annu. Rev. Plant Biol. 55: 373-399.

Aukerman, M.J. and Sakai, H. 2003. Regulation of flowering time and floral organ identity by a microRNA and its APETALA2-like target genes. Plant Cell 15: 2730-2741.
Bartel, D.P. 2004. MicroRNAs: Genomics, biogenesis, mechanism, and function. Cell 116: 281-297.

Carrington, J.C. and Ambros, V. 2003. Role of microRNAs in plant and animal development. Science 301: 336-338.

Cooper, B., Clarke, J.D., Budworth, P., Kreps, J., Hutchison, D., Park, S., Guimil, S., Dunn, M., Luginbuhl, P., Ellero, C., et al. 2003. A network of rice genes associated with stress response and seed development. Proc. Natl. Acad. Sci. 100: 4945-4950.

Dolferus, R., Jacobs, M., Peacock, W.J., and Dennis, E.S. 1994. Differential interactions of promoter elements in stress responses of the Arabidopsis Adh gene. Plant Physiol. 105: 1075-1087.

Eisen, M.B. and Brown, P.O. 1999. DNA arrays for analysis of gene expression. Methods Enzymol. 303: 179-205.

Eisen, M.B., Spellman, P.T., Brown, P.O., and Botstein, D. 1998. Cluster analysis and display of genome-wide expression patterns. Proc. Natl. Acad. Sci. 95: 14863-14868.

Fedoroff, N.V. 2002. Cross-talk in abscisic acid signaling. Sci. STKE 2002: RE10. doi: 10.1126/stke.2002.140.re10.

Fujii, H., Chiou, T.J., Lin, S.I., Aung, K., and Zhu, J.K. 2005. A miRNA involved in phosphate-starvation response in Arabidopsis. Curr. Biol. 15: 2038-2043.

Garzon, R., Pichiorri, F., Palumbo, T., Iuliano, R., Cimmino, A., Aqeilan, R., Volinia, S., Bhatt, D., Alder, H., Marcucci, G., et al. 2006. MicroRNA fingerprints during human megakaryocytopoiesis. Proc. Natl. Acad. Sci. 103: 5078-5083.

Guo, H.S., Xie, Q., Fei, J.F., and Chua, N.H. 2005. MicroRNA directs mRNA cleavage of the transcription factor NAC1 to downregulate auxin signals for Arabidopsis lateral root development. Plant Cell 17: 1376-1386.

Himmelbach, A., Yang, Y., and Grill, E. 2003. Relay and control of abscisic acid signaling. Curr. Opin. Plant Biol. 6: 470-479.

Jaglo-Ottosen, K.R., Gilmour, S.J., Zarka, D.G., Schabenberger, O., and Thomashow, M.F. 1998. Arabidopsis CBF1 overexpression induces COR genes and enhances freezing tolerance. Science 280: 104-106.

Jain, M., Nijhawan, A., Arora, R., Agarwal, P., Ray, S., Sharma, P., Kapoor, S., Tyagi, A.K., and Khurana, J.P. 2007. F-box proteins in rice. Genome-wide analysis, classification, temporal and spatial gene expression during panicle and seed development, and regulation by light and abiotic stress. Plant Physiol. 143: 1467-1483.

Jones-Rhoades, M.W. and Bartel, D.P. 2004. Computational identification of plant microRNAs and their targets, including a stressinduced miRNA. Mol. Cell 14: 787-799.

Kasuga, M., Liu, Q., Miura, S., Yamaguchi-Shinozaki, K., and Shinozaki, K. 1999. Improving plant drought, salt, and freezing tolerance by gene transfer of a single stress-inducible transcription factor. Nat. Biotechnol. 17: 287-291.

Kimura, M., Manabe, K., Abe, T., Yoshida, S., Matsui, M., and Yamamoto, Y.Y. 2003. Analysis of hydrogen peroxide-independent expression of the high-light-inducible ELIP2 gene with the aid of the ELIP2 promoter-luciferase fusions. Photochem. Photobiol. 77: 668-674.

Lescot, M., Dehais, P., Thijs, G., Marchal, K., Moreau, Y., Van de Peer, Y., Rouze, P., and Rombauts, S. 2002. PlantCARE, a database of plant cis-acting regulatory elements and a portal to tools for in silico analysis of promoter sequences. Nucleic Acids Res. 30: 325327. doi: $10.1093 /$ nar/30.1.325.

Leung, A.K. and Sharp, P.A. 2007. MicroRNAs: A safeguard against turmoil? Cell 130: 581-585.

Liu, J., Rivas, F.V., Wohlschlegel, J., Yates 3rd, J.R., Parker, R., and Hannon, G.J. 2005. A role for the P-body component GW182 in microRNA function. Nat. Cell Biol. 7: 1261-1266.

Llave, C., Kasschau, K.D., Rector, M.A., and Carrington, J.C. 2002. Endogenous and silencing-associated small RNAs in plants. Plant Cell 14: 1605-1619.

Lu, S., Sun, Y.H., Shi, R., Clark, C., Li, L., and Chiang, V.L. 2005. Novel and mechanical stress-responsive microRNAs in Populus trichocarpa that are absent from Arabidopsis. Plant Cell 17: 21862203. 
Mallory, A.C., Bartel, D.P., and Bartel, B. 2005. MicroRNA-directed regulation of Arabidopsis AUXIN RESPONSE FACTOR17 is essential for proper development and modulates expression of early auxin response genes. Plant Cell 17: 1360-1375.

Mundy, J., Yamaguchi-Shinozaki, K., and Chua, N.H. 1990. Nuclear proteins bind conserved elements in the abscisic acid-responsive promoter of a rice rab gene. Proc. Natl. Acad. Sci. 87: 1406-1410.

Navarro, L., Dunoyer, P., Jay, F., Arnold, B., Dharmasiri, N., Estelle, M., Voinnet, O., and Jones, J.D. 2006. A plant miRNA contributes to antibacterial resistance by repressing auxin signaling. Science 312: 436-439.

Palatnik, J.F., Allen, E., Wu, X., Schommer, C., Schwab, R., Carrington, J.C., and Weigel, D. 2003. Control of leaf morphogenesis by microRNAs. Nature 425: 257-263.

Park, W., Li, J., Song, R., Messing, J., and Chen, X. 2002. CARPEL FACTORY, a Dicer homolog, and HEN1, a novel protein, act in microRNA metabolism in Arabidopsis thaliana. Curr. Biol. 12: 1484-1495.

Schena, M., Shalon, D., Davis, R.W., and Brown, P.O. 1995. Quantitative monitoring of gene expression patterns with a complementary DNA microarray. Science 270: 467-470.

Sunkar, R., Chinnusamy, V., Zhu, J., and Zhu, J.K. 2007. Small RNAs as big players in plant abiotic stress responses and nutrient deprivation. Trends Plant Sci. 12: 301-309.

Sunkar, R., Kapoor, A., and Zhu, J.K. 2006. Posttranscriptional induction of two $\mathrm{Cu} / \mathrm{Zn}$ superoxide dismutase genes in Arabidopsis is mediated by down-regulation of miR398 and important for oxidative stress tolerance. Plant Cell 18: 2051-2065.

Sunkar, R. and Zhu, J.K. 2004. Novel and stress-regulated microRNAs and other small RNAs from Arabidopsis. Plant Cell 16: 20012019.

Tahbaz, N., Carmichael, J.B., and Hobman, T.C. 2001. GERp95 belongs to a family of signal-transducing proteins and requires
Hsp90 activity for stability and Golgi localization. J. Biol. Chem. 276: 43294-43299.

Thomson, J.M., Parker, J., Perou, C.M., and Hammond, S.M. 2004. A custom microarray platform for analysis of microRNA gene expression. Nat. Med. 1: 47-53.

Tusher, V.G., Tibshirani, R., and Chu, G. 2001. Significance analysis of microarrays applied to the ionizing radiation response. Proc. Natl. Acad. Sci. 98: 5116-5121.

Vaucheret, H., Vazquez, F., Crete, P., and Bartel, D.P. 2004. The action of ARGONAUTE1 in the miRNA pathway and its regulation by the miRNA pathway are crucial for plant development. Genes \& Dev. 18: 1187-1197.

Vazquez, F., Gasciolli, V., Crete, P., and Vaucheret, H. 2004. The nuclear dsRNA binding protein HYL1 is required for microRNA accumulation and plant development, but not posttranscriptional transgene silencing. Curr. Biol. 14: 346-351.

Wu, M.F., Tian, Q., and Reed, J.W. 2006. Arabidopsis microRNA167 controls patterns of ARF6 and ARF8 expression, and regulates both female and male reproduction. Development 133: 4211-4218.

Xu, D., Duan, X., Wang, B., Hong, B., Ho, T., and Wu, R. 1996. Expression of a late embryogenesis abundant protein gene, HVA1, from barley confers tolerance to water deficit and salt stress in transgenic rice. Plant Physiol. 110: 249-257.

Zhang, W., Ruan, J., Ho, T.H., You, Y., Yu, T., and Quatrano, R.S. 2005. Cis-regulatory element based targeted gene finding: Genome-wide identification of abscisic acid- and abiotic stressresponsive genes in Arabidopsis thaliana. Bioinformatics 21: 30743081.

Zhao, B., Liang, R., Ge, L., Li, W., Xiao, H., Lin, H., Ruan, K., and Jin, Y. 2007. Identification of drought-induced microRNAs in rice. Biochem. Biophys. Res. Commun. 354: 585-590.

Zhou, X., Wang, G., and Zhang, W. 2007. UV-B responsive microRNA genes in Arabidopsis thaliana. Mol. Syst. Biol. 3: 103. 

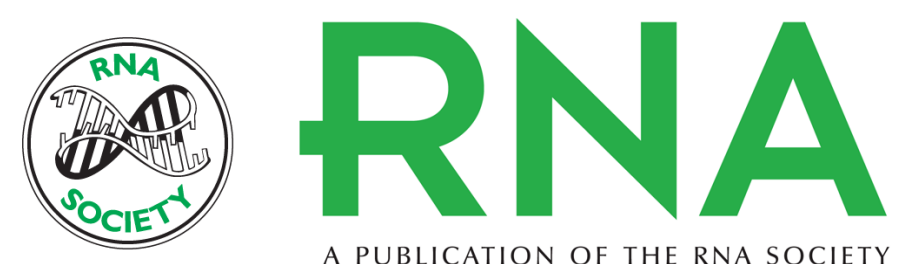

A PUBLICATION OF THE RNA SOCIETY

\section{Microarray-based analysis of stress-regulated microRNAs in Arabidopsis thaliana}

Han-Hua Liu, Xin Tian, Yan-Jie Li, et al.

RNA 2008 14: 836-843

References This article cites 44 articles, 24 of which can be accessed free at:

http://rnajournal.cshlp.org/content/14/5/836.full.html\#ref-list-1

\section{License}

Email Alerting Receive free email alerts when new articles cite this article - sign up in the box at the Service top right corner of the article or click here. 